\title{
Open Globe Injury: Ultrasound First!
}

W e read with much interest the article by Dr. Bodanapally and colleagues ${ }^{1}$ in the March 2017 issue of AJNR regarding the interest of facial CT findings as prognostic predictors of visual outcome in open globe injury. They reported 4 variables associated with poor outcome on a regression model and showed that a grade III posterior segment hemorrhage had a strong positive predictive value of $100 \%$ for profound vision loss. Although these results are interesting and innovative, we believe that the interest in CT as a prognostic tool for visual outcome in open globe injury remains limited.

The exploration of the eyeball is still difficult with CT, even more so in case of injury. That is why the authors chose to refer to posterior eyeball injuries collectively as posterior segment hemorrhage. However, CT is only a mere surrogate for major ocular injuries such as retinal (RD) or choroidal detachments, which are reported to be the most important factors influencing final visual acuity in clinical and surgical studies. ${ }^{2}$ Although vitreous hemorrhage may predict $\mathrm{RD}$, it is not associated with poor visual outcome on its own. From a clinical and practical viewpoint, an emergency surgery is often performed on patients with open globe injuries to explore the eyeball, state the injuries, and perform a vitrectomy in the case of intravitreous hemorrhage. The demonstration of an $\mathrm{RD}$ or choroidal detachment profoundly changes the management of patients because prompt intervention and repair of these injuries significantly improve visual outcomes. $^{2}$ Thus, distinguishing attenuated vitreous membranes from RD is essential. It is, unfortunately, not directly possible with CT.

Moreover, the quantification of posterior segment hemorrhage is difficult with CT because of heterogeneous and varying blood densities in the eyes depending on the location of the hemorrhage, the presence of RD or choroidal detachment, the presence of a hematoma, or the delay between the injury and the CT. The absence of blood visualization on CT is also not reliable because of a lack of sensitivity.

The most appropriate imaging tool to assess prognostic predictors of visual outcome in open globe injury would be sonography. Its role in the detection of RD in eyes with opaque media has

http://dx.doi.org/10.3174/ajnr.A5282 been established clearly in the past. At present, there is no other method to reliably ascertain the anatomic position of the retina when direct examination is impossible. It allows a direct visualization of the main eyeball injuries, such as $\mathrm{RD}$, globe rupture, perforating injury, or endophthalmitis. ${ }^{2}$ These items are used in the main predictive models that have been developed for clinical decision-making, such as the Ocular Trauma Score, ${ }^{3}$ which can predict profound vision loss with a sensitivity of $100 \%$ and specificity of $91 \%$. Sonography has $100 \%$ accuracy in the diagnosis of $\mathrm{RD}$ and can distinguish $\mathrm{RD}$ from choroidal detachments. ${ }^{2}$ It is reported to be more accurate than $\mathrm{CT}$ in the diagnosis of vitreous hemorrhages, ${ }^{4}$ to provide an accurate quantification, and to detect even a small amount of blood that is not visible on CT. It may depict hyphema, lens dislocations, and intraocular foreign bodies. It can provide the exact location of the injuries and can differentiate vitreous from subretinal hemorrhage. It can even indicate some rare injuries that are often inoperable, such as closed-funnel RDs.

Sonography is simple to perform and interpret, easily accessible even at the patient's bedside or in a military setting. It is inexpensive and does not expose patients to ionizing radiation, as opposed to CT. It can be associated with a Doppler measurement of the central retinal artery, which reflects the intraocular hemodynamic, and is also a prognostic predictor for visual outcome. Finally, it is easy to perform sequential sonography during followup, which is crucial because one-quarter of patients with an open globe injury will develop an $\mathrm{RD}$ or a retinal tear after surgical repair. $^{2}$

In conclusion, although interesting in the context of emergency, especially for the detection of ocular foreign bodies, we believe that CT has limited value in immediate clinical decisionmaking and as a prognostic predictor of visual outcome, and we believe that sonography could be a valuable and effective tool to predict outcomes.

\section{REFERENCES}

1. Bodanapally UK, Addis H, Dreizin D, et al. Prognostic predictors of visual outcome in open globe injury: emphasis on facial CT findings. AJNR Am J Neuroradiol 2017;38:1013-18 CrossRef Medline

2. Andreoli MT, Yiu G, Hart L, et al. B-scan ultrasonography following open globe repair. Eye 2014;28:381-85 CrossRef Medline

AJNR Am J Neuroradiol 38:E99-E100 Nov 2017 www.ajnr.org 
3. Kuhn F, Maisiak R, Mann L, et al. The Ocular Trauma Score (OTS). Ophthalmol Clin North Am 2002;15:163-65, vi CrossRef Medline

4. Bäuerle J, Gross NJ, Egger K, et al. Terson's Syndrome: diagnostic comparison of ocular sonography and CT. J Neuroimaging 2016;26: 247-52 CrossRef Medline

(1)A. Lecler

Department of Radiology

(D) A. Pinel

Department of Ophthalmology

(1) P. Koskas

Department of Radiology

Fondation Ophtalmologique Adolphe de Rothschild

Paris, France 